\title{
¿MÁS ALLÁ DEL AÑO DUAL? LA ÚLTIMA DÉCADA DE LAS RELACIONES HISPANO-RUSAS
}

\author{
Javier Morales ${ }^{1}$ \\ UNISCI / St. Antony's College, Universidad de Oxford
}

\begin{abstract}
Resumen:
El objetivo de este artículo es presentar la evolución de las relaciones hispano-rusas desde 2000 hasta la actualidad, que podemos dividir en tres etapas en función de los cambios de gobierno en España — victoria del PSOE en 2004 - y Rusia, con la llegada a la presidencia de Medvedev en 2008. Comenzaremos mencionando brevemente algunos antecedentes históricos, para profundizar después en los ámbitos político, de seguridad, económico y cultural de los contactos bilaterales, y finalizando con unas conclusiones que tratan de definir conceptualmente las principales características de esta relación.
\end{abstract}

Palabras clave: España, Rusia, relaciones bilaterales, Año Dual.

Title in English: "Beyond the Dual Year?: The last Decade of Spain-Russia Relations”.

\begin{abstract}
:
This article presents the evolution of Spanish-Russian relations since 2000, which we can divide into three stages according to the changes of government in Spain - with the Socialist Party's victory in 2004 - and Russia, after Medvedev came to power in 2008. We start with a brief historical overview, and then we continue with an analysis of the political, security, economic and cultural aspects of the bilateral contacts. Finally, in our conclusions we make an attempt at conceptually defining the main features of this relationship.
\end{abstract}

Keywords: Spain, Russia, bilateral relations, Dual Year.

Copyright $@$ C UNISCI, 2011.

Las opiniones expresadas en estos artículos son propias de sus autores, y no reflejan necesariamente la opinión de UNISCI. The views expressed in these articles are those of the authors, and do not necessarily reflect the views of UNISCI.

\footnotetext{
${ }^{1}$ Javier Morales Hernández es Miembro Asociado Senior en el Centro de Estudios Rusos y Eurasiáticos del St. Antony's College, Universidad de Oxford, e Investigador Senior de UNISCI. Sus principales líneas de investigación son la política exterior y de seguridad de Rusia, con especial atención a sus relaciones con Occidente. Dirección: St. Antony’s College, Oxford OX2 6JF, United Kingdom.

E-mail: javier.moraleshernandez@sant.ox.ac.uk. http://dx.doi.org/10.5209/rev_UNIS.2011.v27.38141
} 


\section{Introducción}

El objetivo de este artículo es presentar la evolución de las relaciones hispano-rusas desde 2000 hasta la actualidad, que podemos dividir en tres etapas en función de los cambios de gobierno en España — victoria del PSOE en 2004 - y Rusia, con la llegada a la presidencia de Medvedev en 2008. Comenzaremos mencionando brevemente algunos antecedentes históricos, para profundizar después en los ámbitos político, de seguridad, económico y cultural de los contactos bilaterales, y finalizando con unas conclusiones que tratan de definir conceptualmente las principales características de esta relación.

\section{Antecedentes}

Por motivos históricos, las relaciones entre España y Rusia han sido desiguales desde sus comienzos, alternando etapas de acercamiento y de distanciamiento. En 1667 se envía la primera embajada del zar Alexis Mijailovich a Madrid, pero no se establecerán misiones diplomáticas permanentes hasta 1722. Durante el siglo XVIII se producen varios periodos de ruptura, que se repetirán entre 1918 y 1933 como consecuencia de la instauración del régimen soviético, y nuevamente durante la dictadura franquista hasta la restauración de la democracia en España.

Los antecedentes inmediatos de la etapa actual en los contactos bilaterales se encuentran en los primeros gestos de acercamiento a partir de 1977, tras el restablecimiento de relaciones diplomáticas. Así, ya en 1984 se realiza una visita de los Reyes a Moscú, que se ve sucedida por el viaje de Mijail Gorbachov a Madrid en 1990 y el de Felipe González a Moscú en 1991, aún durante el periodo soviético. Durante esta última visita se firma un tratado bilateral de amistad y cooperación, el cuarto concluido por la entonces URSS con un país occidental tras los establecidos con Alemania, Italia y Francia ${ }^{2}$.

\section{Relaciones políticas}

Las relaciones políticas hispano-rusas en la última década, coincidiendo con el segundo mandato de José María Aznar (2000-2004) y casi la totalidad de las dos presidencias de José Luis Rodríguez Zapatero (desde 2004) — así como con los dos mandatos de Vladimir Putin (2000-2008) y el de Dmitri Medvedev (desde 2008)—, pueden considerarse el periodo de mayor estabilidad en toda la historia de los contactos bilaterales, pese a los momentos de crisis puntuales.

A modo de indicador del clima político entre Madrid y Moscú, podemos observar (fig. 1) que las visitas de alto nivel se han producido con regularidad con independencia de los cambios de gobierno en ambos países. Estos viajes — del rey, el príncipe de Asturias, el presidente del Gobierno o el ministro de Asuntos Exteriores por parte de España, y del presidente, el primer ministro o el ministro de Exteriores por parte rusa- han sido, no obstante, ligeramente más numerosos en cuanto a las personalidades españolas; y menos

\footnotetext{
${ }^{2}$ Ministerio de Asuntos Exteriores y de Cooperación, Dirección General de Comunicación Exterior (2011): Rusia, Madrid, Ministerio de Asuntos Exteriores y de Cooperación, p. 27, en http://www.maec.es; De la Cámara, Manuel: "Las relaciones entre España y la Federación Rusa", CIDOB, Anuario Internacional CIDOB, pp. 450451, en http://www.cidob.org.
} 
frecuentes en los meses anteriores a la celebración de elecciones, por la lógica incertidumbre sobre el impacto de los resultados electorales en los acuerdos que pudieran alcanzarse.

Hay que destacar además la relación personal del rey con dirigentes rusos como Putin, manifestada en visitas privadas además de las oficiales, que han contribuido a facilitar la intensificación de las relaciones bilaterales ${ }^{3}$. Estos contactos pueden haber permitido al monarca ejercer de mediador en determinados momentos, como —-según algunas informaciones - pudo haber ocurrido respecto al interés de la petrolera Lukoil en adquirir una participación en Repsol ${ }^{4}$.

Fig. 1: Visitas de personalidades españolas a Rusia y viceversa, 2000-2010

\begin{tabular}{|c|c|c|c|c|c|c|c|c|c|c|c|c|c|}
\hline & \multirow[t]{2}{*}{2000} & \multirow[t]{2}{*}{2001} & \multirow[t]{2}{*}{2002} & \multirow[t]{2}{*}{2003} & \multicolumn{2}{|c|}{2004} & \multirow[t]{2}{*}{2005} & \multirow[t]{2}{*}{2006} & \multirow[t]{2}{*}{2007} & \multicolumn{2}{|c|}{2008} & \multirow[t]{2}{*}{2009} & \multirow[t]{2}{*}{2010} \\
\hline & & & & & PP & PSOE & & & & Putin & Mdv & & \\
\hline & \multicolumn{5}{|c|}{$A Z N A R$} & \multicolumn{8}{|c|}{$Z A P A T E R O$} \\
\hline Rey & & 1 & 1 & 0 & 0 & 0 & 1 & 1 & 0 & 0 & 1 & 0 & 0 \\
\hline Princ & & 0 & 1 & 1 & 0 & 0 & 0 & 0 & 0 & 0 & 0 & 0 & 0 \\
\hline Pte Gb & & 1 & 0 & 0 & 0 & 1 & 0 & 0 & 1 & 0 & 1 & 1 & 0 \\
\hline MAE & & 1 & 0 & 1 & 0 & 1 & 0 & 1 & 1 & 0 & 1 & 0 & 1 \\
\hline Pte & 1 & 0 & 0 & 0 & 0 & 0 & 0 & 1 & 0 & 0 & 0 & 1 & 0 \\
\hline PM & & 1 & 0 & 0 & 0 & 0 & 0 & 0 & 0 & 0 & 0 & 0 & 0 \\
\hline \multirow[t]{2}{*}{ MAE } & & 0 & 3 & 0 & 0 & 1 & 1 & 0 & 1 & 0 & 0 & 0 & 0 \\
\hline & \multicolumn{5}{|c|}{$P \cup T \mid N$} & & & & & & \multicolumn{3}{|c|}{$M E D V E D E V$} \\
\hline
\end{tabular}

Otra característica de la relación hispano-rusa ha sido la buena disposición por ambas partes, lo que podría interpretarse en dos sentidos. Por un lado, se trataría del resultado de los esfuerzos realizados por ambos gobiernos para superar décadas de desconocimiento y recelos mutuos, y consolidar una cooperación estable que tenga efectos positivos de spillover, desbordando el ámbito político para, a partir de la confianza creada, lograr avances también en otros ámbitos como el económico o el de la seguridad; y pudiendo extenderse igualmente desde el marco bilateral al del conjunto de las relaciones Rusia-UE.

Sin embargo, otra explicación igualmente probable - y no necesariamente incompatible con la anterior - es la de que ha sido, precisamente, la distancia en términos geográficos e históricos ente España y Rusia la que ha hecho posible lograr un clima tan positivo como el actual. La ausencia de conflictos bilaterales, que en muchos casos se derivan de una vecindad geográfica - por ejemplo, la experiencia de sucesivas invasiones y conflictos entre Rusia y Polonia, la situación de las minorías rusas en los países bálticos, o las disputas por el suministro ruso del gas con Ucrania - ha facilitado que los gobernantes españoles y rusos se aproximen a los encuentros bilaterales sin prejuicios tan fuertemente arraigados.

De igual forma, el desconocimiento entre las sociedades civiles hace que la relación con Rusia no sea una preocupación del electorado español — como lo es, por ejemplo, para el

\footnotetext{
${ }^{3}$ Por ejemplo: "Putin y el Rey Don Juan Carlos destacan el buen momento de las relaciones Rusia-España", La Vanguardia, 18 de agosto de 2002, en http://www.lavanguardia.com.

4 "El rey llamó a Zapatero seis veces para apoyar a Lukoil", Público, 26 de noviembre de 2008, en http://www.público.es.

5 Elaboración propia a partir de datos del Ministerio de Asuntos Exteriores y de Cooperación (2000-2010): Anuario de Política Exterior, en http://www.maec.es.
} 
alemán—, y mucho menos para los ciudadanos rusos en cuanto a la relación con España. Esto otorga un amplio margen de actuación a los dirigentes para actuar de forma pragmática, sin verse constreñidos por los compromisos ideológicos adquiridos ante sus votantes.

Todos estos factores hacen que las relaciones hispano-rusas tiendan a ser "positivas por defecto", de forma que son otros actores o polos de atracción externos los que determinan los momentos de mayor cercanía o distanciamiento entre nuestros dos países. Así, por ejemplo, el reinicio o reset entre Washington y Moscú tras la llegada de Obama a la presidencia ha sido indudablemente un factor positivo — entre otros — que ha impulsado una mayor atención del gobierno de Zapatero hacia Rusia, como veremos a continuación. Del mismo modo, los desacuerdos con la UE a raíz de las "crisis del gas" ruso-ucranianas se extendieron a España, creando un clima político de rechazo a la participación de empresas rusas en el sector energético español.

Una consecuencia adicional de esta tendencia a definir las relaciones bilaterales a partir de la inercia creada por la ausencia de conflictos es que, de forma creciente, se tiende a dar prioridad a los ámbitos con una menor carga política y de carácter más pragmático, como el económico - donde los intereses son en gran medida complementarios- y el cultural, que representa el ejemplo más claro de "juego de suma positiva" sin recelos por ninguna de ambas partes. Pese a que estos avances tienen sin duda el efecto de spillover que hemos mencionado, creando un conocimiento y confianza mutuas que se extienden al diálogo político, por sí solos no pueden sostener una cooperación estable en el tiempo, en ausencia de un impulso decidido al máximo nivel de considerarse socios también en el terreno de la política internacional, tratando de acercar posiciones en temas donde los intereses no son tan evidentemente similares.

Las relaciones hispano-rusas en esta última década se pueden dividir, como hemos señalado, en tres etapas determinadas por los respectivos cambios de gobierno.

\subsection{La etapa Aznar-Putin (2000-2004)}

En el periodo 2000-2004, con Aznar y Putin al frente de los ejecutivos español y ruso, el polo de atracción externo más relevante para los contactos bilaterales puede considerarse EE.UU. Es la reacción de la Administración Bush a los atentados del 11-S, con la invasión de Afganistán y posteriormente de Irak, la que marca las tendencias en las relaciones de Rusia con Occidente, incluyendo a España. Las consecuencias positivas de esto son un impulso a la cooperación bilateral en el ámbito de la seguridad, no sólo en cuanto a la lucha contra el terrorismo de inspiración islamista radical, sino también en otras cuestiones como el crimen organizado.

Asimismo, tanto Rusia como España apoyan el derrocamiento del régimen de los talibanes en Afganistán, e incluso las diferencias posteriores en relación con la guerra de Irak permiten - gracias al pragmatismo mostrado por ambas partes - que no se deteriore la cooperación en otros ámbitos. Son otros acontecimientos, como la retórica estadounidense de "extensión de la democracia" y las "revoluciones de colores" en Ucrania o Georgia, lo que hace que Rusia considere las políticas de Washington como una amenaza política directa para la estabilidad de su régimen. De esta forma, pese a estos ejemplos prácticos de colaboración en áreas de interés común, la confianza en el terreno político se ve reducida como consecuencia de la actuación de un tercer actor, EE.UU., con el que el gobierno Aznar se identifica de forma abierta. 
Otro factor negativo es que, debido a la asimetría en cuanto al peso específico o poder en el sentido tradicional entre Rusia y España, es Moscú quien tiene menos incentivos para el diálogo con nuestro país si éste no le aporta ventajas que no puede lograr en otros marcos bilaterales, como el que tiene con otros países europeos. Por esta razón, la cercanía de nuestro gobierno a las posiciones de la Administración Bush restaba interés a los dirigentes rusos a la hora de considerar a España un interlocutor diferenciado, centrando la atención en el diálogo entre Washington y Moscú.

Del mismo modo, pese a que España considera positiva la relación con Rusia en cuestiones específicas como las ya mencionadas, el diseño de la estrategia general de política exterior en clave atlantista excluye a Rusia de nuestras áreas prioritarias, relegándola al papel de actor externo al espacio euratlántico y, en el peor de los casos, al de rival para instituciones como la OTAN. Esta visión del mundo en clave de "suma cero", tomada directamente de la ideología neoconservadora estadounidense, tiene sin duda — por la herencia de la Guerra Fría- su equivalente dentro de la propia Rusia; lo que impide que la cooperación llegue a ser profunda y estable incluso en aquellas áreas más técnicas o de menor carga política.

\subsection{La etapa Zapatero-Putin (2004-2008)}

Tras la llegada de Zapatero a la presidencia, no se produce inmediatamente un aumento de la prioridad asignada a Rusia como una de las áreas de nuestra política exterior. Por el contrario, la reorientación llevada a cabo por el gobierno socialista situó a la Unión Europea - y en el marco bilateral, a Estados europeos como Francia o Alemania- como el principal polo de atracción externo y medio a través del cual ejercer una influencia en los asuntos internacionales. Esta visión permite, no obstante, una aproximación más similar a la rusa por su defensa de un mundo multipolar, donde los papeles de la UE y Rusia como potencias sean compatibles entre sí, sin definirse necesariamente en función de su afinidad con una superpotencia con pretensiones hegemónicas.

Del mismo modo, el "retorno a Europa" planteado por Zapatero sitúa a nuestro país en un escenario, como el europeo, donde Rusia desea contar con interlocutores entre los grandes países la UE: principalmente Francia y Alemania — con los que Moscú celebra incluso varias cumbres tripartitas - , pero también Italia por los intereses económicos y la amistad personal entre Berlusconi y Putin. España puede así aprovechar sus buenas relaciones con el "eje franco-alemán" para sumarse al núcleo de países europeos con unos contactos más fluidos con Rusia; si bien no cuenta, naturalmente, con la misma relevancia para Moscú que la de Berlín, París o Roma. Esto marca una diferencia con la etapa anterior, en la que EE.UU. y Rusia definían sus relaciones mediante el diálogo bilateral, sin dar oportunidades a una España alineada con Washington de influir en ellas.

No obstante, el menor peso específico de España en comparación con las grandes potencias europeas impide aprovechar plenamente esta posición como miembro de la UE para asumir un papel más activo en la definición de la política europea hacia Rusia. Por otra parte, hay que reconocer que no se trata sólo de una carencia de capacidades sino también de voluntad política: nuestra diplomacia continúa volcada en las "áreas tradicionales" de Europa, el Magreb-Oriente Medio y Latinoamérica, a las que se suma una incipiente presencia en Asia-Pacífico. El espacio postsoviético es todavía en este momento un escenario alejado de las preocupaciones de nuestros responsables políticos de uno u otro signo, lo cual impide realizar grandes avances más allá de la inercia creada por la limitada cooperación en áreas de bajo contenido político. 


\subsection{La etapa Zapatero-Medvedev (desde 2008)}

El relevo en el Kremlin de Putin por Medvedev facilita un cierto "deshielo" en las relaciones entre Rusia y Occidente, especialmente una vez Barack Obama asume la presidencia de EE.UU. Al mismo tiempo, la UE se plantea recuperar la confianza perdida en las relaciones ruso-europeas, tratando de solucionar las crisis que se produzcan de forma puntual sin que esto implique una paralización del diálogo. Esto permite, por ejemplo, que la crisis ocasionada por la guerra ruso-georgiana de agosto de 2008 se resuelva rápidamente gracias a la mediación europea durante la presidencia francesa; y que en lugar de abrir una nueva etapa de enfrentamientos se reafirme la necesidad de superar los problemas existentes. EE.UU. y la UE serán así los polos de atracción hasta hoy; condicionando con su paralelo restablecimiento de relaciones estables con Moscú las iniciativas en el mismo sentido del gobierno español.

España asiste a esta restauración de las relaciones Rusia-Occidente desde una posición ciertamente más activa que en etapas anteriores. En primer lugar, en marzo de 2009 tiene lugar una visita de Estado de Medvedev a España, durante la cual se firma una declaración de "Asociación Estratégica"6, convirtiéndonos así en el cuarto miembro de la UE - tras Alemania, Francia e Italia- con esta especial relación con Rusia. Las iniciativas propuestas (fig. 2) comprenden desde áreas con mayor carga política, como las consultas e incluso coordinación de las respectivas políticas exteriores en asuntos de interés común, hasta otras más técnicas o menos controvertidas, como la cooperación en educación o cultura.

En segundo lugar, España ocupó la presidencia de turno de la UE en el primer semestre de 2010, incluyendo la intensificación de las relaciones con Rusia como uno de los objetivos de la misma: así, Zapatero llegó a afirmar que "Rusia es un gran aliado, imprescindible para la UE", y que la estabilidad de la Unión "depende en buena medida de las relaciones con Rusia", . Se nombró una Embajadora en Misión Especial para las relaciones con Rusia durante el semestre de presidencia, María Victoria Scola, y se plantearon como objetivos los siguientes: revisión del sistema de visados, participación rusa en determinados programas de la Asociación Oriental, cooperación energética, apoyo al ingreso de Rusia en la Organización Mundial de Comercio (OMC) - con vistas a un posible acuerdo de libre comercio en el futuro-, y lucha contra el terrorismo, el crimen organizado y el narcotráfico ${ }^{8}$.

\footnotetext{
${ }^{6}$ Declaración de Asociación Estratégica entre el Reino de España y la Federación de Rusia, 3 de marzo de 2009, en http://www.la-moncloa.es.

7 “Zapatero apuesta por acercar Rusia a la UE para garantizar la seguridad”, La Vanguardia, 13 de abril de 2010, en http://www.lavanguardia.es.

${ }^{8}$ Véase Morales Hernández, Javier: "Las relaciones UE-Rusia durante la presidencia española", en Marquina, Antonio (ed.) (2010): La política exterior de seguridad y defensa común de la Unión Europea II: resultados de la presidencia española, UNISCI Papers no 36 , Madrid, UNISCI.
} 
Fig. 2: Contenido de la Asociación Estratégica España-Rusia (2009) ${ }^{9}$

\begin{tabular}{|c|c|c|}
\hline & ÁMBITO & ACCIONES DE COOPERACIÓN PROPUESTAS \\
\hline & Política exterior & $\begin{array}{ll}\text { - } & \text { Información mutua, coordinación e iniciativas conjuntas en asuntos de } \\
\text { interés común } \\
\text { - } & \text { Consultas anuales entre ministros de AA.EE. } \\
\text { - } & \text { Negociación de un nuevo Tratado de Amistad y Cooperación }\end{array}$ \\
\hline 2. & $\begin{array}{l}\text { Seguridad y } \\
\text { Justicia }\end{array}$ & $\begin{array}{ll}\text { - } & \text { Colaboración entre Ministerios de Defensa / FAS } \\
\text { - } & \text { Convenio sobre tránsito de equipos y personal españoles por territorio ruso } \\
\text { - } & \text { hacia Afganistán } \\
\text { - } & \text { Lucha contra el terrorismo contra la proliferación de armas de destrucción masiva } \\
\text { - } & \text { Lucha contra el crimen organizado } \\
\text { - } & \text { Lucha contra el tráfico de drogas } \\
\text { - } & \text { Reacción ante catástrofes naturales o de origen humano } \\
\text { - } & \text { Colaboración entre organismos jurídicos (Memorando de Entendimiento } \\
& \text { entre Fiscalías Generales) }\end{array}$ \\
\hline 3. & $\begin{array}{l}\text { Economía y } \\
\text { Comercio }\end{array}$ & $\begin{array}{ll}\text { - } & \text { Fomento del comercio } \\
\text { - } & \text { Fomento del turismo (Programa de Acciones Conjuntas) } \\
\text { - } & \text { Fomento de las inversiones y protección recíproca } \\
\text { - } & \text { Intercambio de información económica } \\
\text { - } & \text { Contactos entre empresas y cámaras de comercio } \\
\text { - } & \text { Cooperación en infraestructuras y equipamientos (incluyendo Protocolo de } \\
& \text { Cooperación ferroviaria) } \\
\text { - Cooperación energética: petróleo, gas, carbón y renovables (Memorando } \\
\text { - Co Entendimiento) } \\
\text { - Cooperación en I+D+i, especialmente en energía nuclear, tecnología } \\
\text { - Facilitar ingreso de Rusia en la OMC }\end{array}$ \\
\hline & $\begin{array}{l}\text { Cultura y } \\
\text { Sociedad }\end{array}$ & $\begin{array}{l}\text { - } \quad \text { Relaciones entre universidades, centros docentes y de investigación } \\
\text { - } \quad \text { Fomento de la movilidad de profesores e investigadores } \\
\text { - } \quad \text { Colaboración entre instituciones e industrias culturales gubernamentales y } \\
\text { - } \quad \text { Co gubernamentales, impulsando actividades de cada país en el otro } \\
\text { - } \quad \text { Protección de la propiedad intelectual } \\
\text { - } \quad \text { Digitalización e intercambio de archivos } \\
\text { - } \quad \text { Foro Hispano-Ruso de Diálogo entre Sociedades Civiles (anual) } \\
\text { - Colaboración consular, aplicación favorable del régimen de visados y } \\
\text { - Diálogo para futura supresión }\end{array}$ \\
\hline
\end{tabular}

El resultado más claro del semestre en cuanto a las relaciones eurorusas fue la firma de la llamada "Asociación para la Modernización" en la cumbre celebrada en Rostov del Don. Como se afirmaba en la declaración conjunta de dicha cumbre ${ }^{10}$, Rusia y la UE se consideran mutuamente como "socios estratégicos", y basarán esta nueva asociación en el fomento de la inversión, el comercio bilateral y las relaciones económicas; la aproximación de los estándares y normativas técnicas, y los derechos de propiedad intelectual; la mejora de los transportes; la promoción de una economía sostenible, eficiencia energética y lucha contra el

\footnotetext{
${ }^{9}$ Morales Hernández, Javier (2010): "España y Rusia ante el año bilateral: oportunidades para 2011”, Instituto Español de Estudios Estratégicos, Documento de Opinión del IEEE 21/2010, , p. 3.

${ }^{10}$ Council of the European Union: "Joint Statement on the Partnership for Modernisation. EU-Russia Summit", 1 de junio de 2010, en http://www.consilium.europa.eu.
} 
cambio climático; la cooperación en investigación, desarrollo e innovación en estas áreas, así como en otras como la tecnología espacial.

El objetivo principal de Rusia era conseguir la cooperación de la UE para su desarrollo tecnológico, mediante proyectos conjuntos - como las energías alternativas o el aumento de la eficiencia energética- que permitan alcanzar resultados con un presupuesto moderado, debido al actual contexto de crisis; así como la adopción de estándares comunes e incluso de legislación comunitaria, fomento de las inversiones e intercambios académicos y de profesionales altamente cualificados ${ }^{11}$. La respuesta de la UE, por su parte, se centró en señalar la necesidad de unir la modernización política a la económica, avanzando en la democratización de Rusia, combatiendo la corrupción, reduciendo las restricciones a los inversores extranjeros y permitiendo la implicación de la sociedad civil ${ }^{12}$.

\section{Relaciones de seguridad}

Las relaciones hispano-rusas en el ámbito de la seguridad se definen, como ya hemos señalado en cuanto a la cooperación política, por la ausencia de percepciones mutuas de amenaza. En cambio, existen intereses compartidos en cuanto a la lucha contra el terrorismo y el crimen organizado, que han dado lugar a una cooperación frecuente y fluida entre las respectivas fiscalías generales - con un Memorando de Entendimiento firmado durante la visita de Medvedev en 2009-, policías y servicios de inteligencia, como se resalta habitualmente por ambas partes.

Pese a ello, esta cooperación puede no ser suficiente en algunos aspectos: por ejemplo, un fiscal de la Audiencia Nacional especializado en el crimen organizado de la antigua URSS —según reveló uno de los cables secretos publicados por Wikileaks ${ }^{13}$ - enfatizaba las dimensiones de este fenómeno y sugería que los propios servicios de seguridad rusos mantienen relaciones con este tipo de grupos, lo cual dificulta la persecución de criminales rusos en su país por delitos cometidos en España. Otro aspecto problemático, esta vez desde el punto de vista de Moscú, es el hecho de que los medios de comunicación españoles utilicen con frecuencia el término "mafia rusa" para referirse a bandas originarias de cualquier país de Europa Central y Oriental o el espacio postsoviético; lo cual perjudica injustificadamente la imagen colectiva de los rusos residentes en España.

Con respecto a la seguridad internacional, se alcanzó un acuerdo para el tránsito por territorio ruso de personal militar español con destino a Afganistán; pero no se han realizado avances sustanciales en otros aspectos, ya que la iniciativa permanece en manos de otros foros - como el Consejo OTAN-Rusia_ más que en el plano bilateral hispano-ruso. En el marco de la UE, el acuerdo con Rusia sobre protección recíproca de información clasificada alcanzado tras la presidencia española de 2010 sirvió para facilitar la continuación de la cooperación eurorusa en misiones — de la UE o conjuntas en el futuro-, ya demostrada en EUFOR Chad/RCA o en las patrullas contra la piratería dentro de la operación Atalanta.

\footnotetext{
11 "Programma effektivnogo ispolzovaniya na sistemnoy osnove vneshnepoliticheskij faktorov v tselyaj dolgosrochnogo razvitiya Rossiyskoy Federatsii", Russkiy Newsweek, 11 de mayo de 2010, en http://www.runewsweek.ru.

12 "EC suggests a modernization plan to Russia", RT, 11 de febrero de 2010, en http://rt.com.

13 "10MADRID154, Spain Details its Strategy to Combat the Russian Mafia", 8 de febrero de 2010, en http://wikileaks.org.
} 
Más recientemente, la intervención de la coalición occidental —incluida España— en la guerra civil de Libia ha demostrado tanto las posibilidades como las limitaciones de la asociación estratégica hispano-rusa. Este conflicto supone probablemente el primer gran asunto de relevancia internacional desde la guerra de Irak en el que Moscú y Madrid han adoptado inicialmente posiciones opuestas; sin embargo, no se ha producido un deterioro apreciable de la confianza entre ambas partes. El pragmatismo para evitar que desacuerdos puntuales dañen el estado de las relaciones es así la tónica general, de igual forma que Moscú no vetó la resolución del Consejo de Seguridad de Naciones Unidas sobre Libia aunque después mostrase sus dudas sobre el grado de intervencionismo de la coalición, y terminase por reconocer finalmente a los sublevados como autoridad legítima del país.

La anunciada colaboración española en la defensa antimisiles de la OTAN, que contará con buques estadounidenses dotados del sistema Aegis en la base naval de Rota, ha creado un punto de fricción en las relaciones hispano-rusas. El proyecto de defensa antimisiles fue precisamente una de las áreas de cooperación en las que Rusia y la OTAN se plantearon colaborar a partir de la cumbre de Lisboa en 2010. No obstante, mientras que Moscú desearía un sistema totalmente conjunto, la Alianza no desea depender de los intereses rusos a la hora de afrontar posibles amenazas con misiles. Este nuevo paso supone la constatación de que EE.UU. está decidido a seguir adelante con el "escudo antimisiles", colaborando con aquellos socios o aliados que lo deseen - como España en este caso-, pero sin someterse a un proceso de consultas con países como Rusia que pueda paralizar el proyecto ${ }^{14}$.

Otras cuestiones de seguridad que han podido dificultar en alguna medida las relaciones son las actividades de los respectivos servicios de inteligencia: así, a finales de 2010 se expulsó a dos diplomáticos españoles acreditados en Moscú como represalia por la expulsión anterior de dos diplomáticos rusos por "actividades incompatibles con su estatus"15. No obstante, tampoco estos incidentes han supuesto un bloqueo de la cooperación ni del impulso al Año Dual que se está celebrando en 2011, y que trataremos más adelante.

\section{Relaciones económicas}

La principal motivación para esta atención renovada por parte española a los contactos con Rusia es indudablemente de carácter económico, especialmente en el actual contexto de crisis. Es frecuente la afirmación de que la intensidad de las relaciones comerciales entre nuestros dos países está muy por debajo del nivel de las relaciones políticas, y que esto supone un obstáculo que es necesario resolver. Los intercambios todavía dan lugar a una balanza comercial negativa para nuestro país (fig. 2): importamos fundamentalmente materias primas -como el hierro, el acero o el níquel— ${ }^{16}$, pero exportamos productos de valor tecnológico como los automóviles, junto con otros como los alimentos (fig. 3).

\footnotetext{
${ }^{14}$ Kamp, Karl Heinz: "Missile Defence - A Sticking Point?", The World Today, vol. 67, no 10 (octubre 2011), pp. 21-23; "EE UU desplegará en Rota 1.100 militares y cuatro buques del escudo antimisiles", El País, 5 de octubre de 2011, en http://www.elpais.com; Vega, Enrique: "Seguridad y juego político", Público, 12 de octubre de 2011, en http://www.publico.es.

${ }^{15}$ Ministerio de Asuntos Exteriores y de Cooperación, "Rusia..., op. cit., p. 33.

${ }^{16}$ De la Cámara, op. cit., p. 452.
} 
Fig. 2: Comercio España-Rusia (mill. Euros) $)^{17}$

$\begin{array}{lrrrr}\text { Balanza Comercial } & \mathbf{2 0 0 7} & \mathbf{2 0 0 8} & \mathbf{2 0 0 9} & \mathbf{2 0 1 0} \\ \text { Importaciones } & 7.892,71 & 7.531,95 & 4.587,22 & 6.125,52 \\ \text { Exportaciones } & 2.085,93 & 2.835,63 & 1.477,43 & 1.994,97 \\ \text { Saldo } & -5.806,78 & -4.696,32 & -3.109,79 & -4.130,55 \\ \text { Tasa de cobertura } & 26,40 & 37,60 & 32,20 & 32,50 \\ \text { \% Variación Importación * } & 7,49 & -4,57 & -39,10 & 33,53 \\ \text { \% Variación Exportación * } & 38,17 & 35,94 & -27,90 & 35,03 \\ \text { (*) Tasa variación sobre año anterior. } & & & & \end{array}$

Fig. 3: Distribución del comercio por productos $(2010)^{18}$

\begin{tabular}{|c|c|c|}
\hline Principales productos importados & Importe & $\%$ del total \\
\hline Combustibles minerales, aceites minerales & $5.343,84$ & 87,20 \\
\hline Fundición, hierro y acero & 371,10 & 6,00 \\
\hline Niquel en bruto & 132,21 & 2,10 \\
\hline Caucho sintético y caucho facticio deriv. de aceite & es 48,80 & 0,70 \\
\hline Abonos & 40,39 & 0,60 \\
\hline Aluminio y manufacturas de aluminio & 21,58 & 0,30 \\
\hline Total partidas & $5.957,92$ & 96,90 \\
\hline Total importaciones & $6.125,52$ & 100,0 \\
\hline Principales productos exportados & mporte & $\%$ del total \\
\hline Calder & 252,69 & 12,60 \\
\hline automóviles, tractores, ciclos y demás & 247,76 & 12,40 \\
\hline Carnes y despojos comestibles & 132,92 & 6,60 \\
\hline Frutos comestibles; cortezas de agrios o de melo & on. 116,00 & 5,80 \\
\hline Baldosas y losas de cerámica para pavimiento & 102,56 & 5,60 \\
\hline Máquinas, aparatos y material eléctrico & 91,49 & 4,50 \\
\hline Total partidas & 943,42 & 47,50 \\
\hline Total exportaciones & $1.994,97$ & 100,00 \\
\hline
\end{tabular}

Dentro del sector energético, Rusia es nuestro principal proveedor de petróleo (16\%) aunque en un mercado muy diversificado: le siguen Irán (14,8\%), Arabia Saudí $(13,7 \%)$, Nigeria $(13,4 \%)$ o México $(11,9 \%)$, entre otros. Con respecto al gas, donde la dependencia de pocos suministradores suele ser más frecuente por la configuración de los gasoductos, España no importa actualmente gas de Rusia ${ }^{19}$. Sin embargo, la posibilidad de que el Foro de Países Exportadores de Gas (GECF) pudiera convertirse en el futuro, con el impulso de miembros como Rusia e Irán, en un verdadero "cártel del gas" similar a la OPEP podría perjudicar los

\footnotetext{
${ }^{17}$ Ministerio de Asuntos Exteriores y de Cooperación, "Rusia..., op. cit., p. 34.

18 Ibid.

${ }^{19}$ Boletín CORES, no 164 (julio 2011), en http://www.cores.es.
} 
intereses españoles; ya que nuestros proveedores - Argelia principalmente, pero también otros como Nigeria o Qatar-participan en esta organización ${ }^{20}$.

El efecto de la crisis ha contribuido a reducir aún más las exportaciones españolas a Rusia, que sólo suponen un $1,3 \%$ de sus importaciones ${ }^{21}$; sólo unas 100 empresas españolas tienen presencia en ese país — por ejemplo, Técnicas Reunidas, Iberdrola, Uralita, Roca, Gallina Blanca o Inditex-, en comparación con 3.000 alemanas. Son todavía muy escasas las compañías españolas que han conseguido proyectos de envergadura en el mercado ruso: por ejemplo, la construcción de una refinería en Jabarovsk por Técnicas Reunidas, con un valor de 800 millones de dólares ${ }^{22}$.

Por parte rusa existe un interés en la participación española en sectores como las infraestructuras —especialmente construcción de centrales energéticas o de ferrocarriles-, el desarrollo de las energías renovables y la lucha contra el cambio climático, recogidos en los acuerdos específicos firmados durante la visita de Medvedev. Para facilitarlo existe una Comisión Mixta de Cooperación Económica e Industrial con representantes de ambos gobiernos, con distintas comisiones de trabajo sobre cambio climático, construcción naval, espacio, turismo, infraestructuras de transporte y otras, y energía ${ }^{23}$.

Esta economización de las relaciones con Rusia no es exclusiva del ámbito bilateral español, sino que también la UE concede una creciente prioridad a estas cuestiones como forma de facilitar una aproximación de los intereses que repercuta positivamente en otras áreas. Así, en la Asociación para la Modernización establecida en la cumbre UE-Rusia de Rostov del Don, al final de la presidencia española de 2010, se recoge una serie de áreas de marcado carácter económico ${ }^{24}$ :

- aumentar las oportunidades para la inversión, el comercio bilateral y las relaciones económicas;

- aproximar los estándares y normativas técnicas, y proteger los derechos de propiedad intelectual;

- mejorar el transporte;

- promover una economía sostenible, la eficiencia energética y la lucha contra el cambio impacto climático;

- reforzar la cooperación en investigación, desarrollo e innovación, incluyendo la tecnología espacial;

- reducir el impacto social negativo de las reestructuraciones económicas;

- garantizar el funcionamiento eficaz del sistema judicial y la lucha contra la corrupción;

\footnotetext{
${ }^{20}$ Gas Exporting Countries Forum: http://www.gecforum.org.

${ }^{21}$ Instituto Español de Comercio Exterior (ICEX): http://www.icex.es.

${ }^{22}$ Sebastián de Erice, José Pedro: Ponencia presentada en el Seminario Rusia-Unión Europea

"El mundo, visto desde Rusia, Asociación de Periodistas Europeos", Madrid, 14 de mayo de 2010.

${ }^{23}$ De la Cámara, op. cit., p. 453.

${ }^{24}$ Council of the European Union: "Joint Statement on the Partnership for Modernisation. EU-Russia Summit", 1 de junio de 2010, en http://www.consilium.europa.eu.
} 
- promover los contactos entre personas y el diálogo con la sociedad civil, facilitando la participación de ciudadanos y empresarios.

España inició también durante su presidencia un diálogo UE-Rusia para la flexibilización del régimen de visados, con la posibilidad de una futura supresión si se llegase a alcanzar un consenso; y de forma recíproca a una futura medida similar por parte de Moscú, que ha venido reclamando durante mucho tiempo la libre circulación de sus ciudadanos en la $\mathrm{UE}^{25}$. Si se llegasen a solventar los obstáculos técnicos, esto tendría un impacto muy positivo para aumentar el conocimiento entre las sociedades civiles, aumentar los contactos empresariales y el turismo - España es ya uno de los principales destinos para los turistas rusos ${ }^{26}$, lo que podría incrementarse aún más facilitando los visados o suprimiéndolos, como han hecho ya Turquía, Israel, Egipto o Túnez-, y reducir a largo plazo la desconfianza entre ambas partes. Rusia acogió muy positivamente esta propuesta, contribuyendo por su parte a flexibilizar la concesión de visados de negocios a ciudadanos europeos ${ }^{27}$.

En los últimos meses, dentro de las medidas de estímulo a la economía española, se han realizado además varias acciones como el lanzamiento de un plan "Made in/by Spain" para mejorar la imagen de las empresas españolas en Rusia, un acuerdo de protección de patentes españolas en este país — facilitando así la exportación de tecnología_, o la visita de la secretaria de Estado de Vivienda, Beatriz Corredor, a Moscú para promocionar el mercado inmobiliario español, en el que los compradores rusos aumentaron un 20\% durante 2010 respecto al año anterior ${ }^{28}$.

El acontecimiento más destacado ha sido el viaje de Zapatero en junio de 2011 para intervenir en el Foro Económico de San Petersburgo, donde España era el país invitado de esta edición. Se celebró una reunión de ambos presidentes con participación de las principales empresas españolas con intereses en Rusia — por ejemplo, Iberdrola, Repsol, Talgo, Roca o Técnicas Reunidas-, y se firmaron veinte acuerdos por valor de 800 millones de euros ${ }^{29}$. Pese a estos esfuerzos, se han producido no obstante algunas crisis puntuales en las relaciones comerciales: por ejemplo el cierre del mercado ruso a las exportaciones españolas durante la "crisis del pepino" causada por el brote de e.coli, que en un principio se atribuyó erróneamente a nuestros productos.

\section{Relaciones culturales}

El principal déficit de la asociación estratégica hispano-rusa ha sido su falta de visibilidad de cara a la opinión pública, más allá de la reducida comunidad de expertos en relaciones

\footnotetext{
25 "News Conference following EU-Russia Summit", 1 de junio de 2010, en http://eng.news.kremlin.ru: Von Twickel, Nicolaus: "Russians Put Visas on EU Summit Agenda", The Moscow Times, 21 de mayo de 2010, en http://www.themoscowtimes.com.

${ }^{26}$ El consulado español en Moscú es el que más visados expide de todos los consulados españoles en el extranjero. De la Cámara, op. cit., p. 454.

27 "Speech at St Petersburg International Economic Forum Plenary Session", 18 de junio de 2010, en http://eng.kremlin.ru

${ }^{28}$ Ministerio de Asuntos Exteriores y de Cooperación, "Rusia..., op. cit., p. 32; "Beatriz Corredor explica en Rusia el potencial del mercado inmobiliario español", 8 de junio de 2011, en http://www.lamoncloa.gob.es.

29 "Rodríguez Zapatero: "Exportar es la forma de acelerar la recuperación y crear empleo", 18 de junio de 2011 , en http://www.lamoncloa.gob.es.
} 
internacionales. Mientras que entre los rusos — pese a la desproporción existente en cuanto al tamaño y población de ambos países- España es apreciada por el atractivo de su idioma, cultura e historia, no sucede en absoluto lo mismo a la inversa. Rusia continúa siendo la más desconocida de nuestros vecinos europeos para una sociedad española que, en cambio, ha aprendido a considerar como propios los problemas que afectan a nuestros socios de la UE. El aprendizaje del ruso - lingua franca en todo el espacio postsoviético- es aún una vocación minoritaria entre los españoles, mientras que por parte rusa el castellano es uno de los idiomas más estudiados. Ejemplo de ello es el Instituto Cervantes de Moscú, que con 80.000 alumnos cada año es el mayor centro de enseñanza de nuestro idioma en todo el mundo ${ }^{30}$.

Para contribuir a aumentar este conocimiento, se está realizando durante 2011 el "Año de Rusia en España", el cual tiene su contrapartida en el "Año de España en Rusia" que se celebra simultáneamente. El "Año Dual"31, como se ha denominado, tiene un contenido marcadamente cultural, con exposiciones, conciertos y conferencias; aunque también se incluyen encuentros de empresarios. Otras iniciativas han tratado el fomento de la movilidad de estudiantes y científicos; se han celebrado también encuentros de rectores de universidades de Rusia y el espacio iberoamericano. Sin embargo, estas actividades por sí solas pueden quedar desconectadas del "núcleo duro" de la política exterior —es decir, la cooperación política ante los desafíos para la estabilidad internacional-, centrándose en las relaciones culturales y en cierta medida también las económicas, por ser éstas, precisamente, aquéllas en las que los intereses de ambas partes están más próximos y se benefician mutuamente.

Al mismo tiempo, el "Año Dual" ha coincidido con distintas circunstancias internas e internacionales que han desplazado la prioridad hacia otros asuntos, de forma que precisamente durante 2011 la visibilidad de las relaciones políticas bilaterales está siendo quizás inferior a la de otros momentos. Por ejemplo, además del notable impacto de la crisis económica, los debates en los últimos meses sobre la sucesión de Zapatero y la candidatura o no de Medvedev a las elecciones del próximo año — pese a las evidentes diferencias entre el sistema político y los equilibrios de poder en ambos países- han contribuido a centrar la agenda en cuestiones internas con más frecuencia de la deseada.

\section{Conclusiones}

El pragmatismo orientado hacia la cooperación económica y libre de condicionamientos ideológicos es la característica principal de las relaciones entre Rusia y España, facilitadas por la ausencia de conflictos - "positivas por defecto", como las definíamos al comienzo-, pero también dificultadas precisamente por el desconocimiento que aún existe por ambas partes, tanto entre los gobernantes como entre las sociedades civiles.

La inercia de las "áreas tradicionales" de la diplomacia española, que no se ha adaptado suficientemente al mundo globalizado y al papel que nuestro país puede desempeñar en él, permite que nuestras políticas hacia Rusia oscilen en función de las iniciativas de otros actores, sin una apuesta clara por consolidarlas como una prioridad para España. La economización de las relaciones a la que nos hemos referido supone un paso positivo, en cuanto que identifica intereses concretos para España que nos impulsan a fomentar los

\footnotetext{
${ }^{30}$ http://moscu.cervantes.es/es/default.shtm

31 http://www.spain-russia2011.ru/
} 
contactos bilaterales; pero también nos limitan a aquellas cuestiones más técnicas y que evitan pronunciarnos sobre una definición política que dote de contenido a la asociación estratégica.

En cuanto al esperado retorno de Putin al Kremlin tras las elecciones que van a celebrarse en 2012, la primera impresión podría hacer temer un retroceso de la cooperación alcanzada si Rusia adopta una posición más distanciada de Occidente, incluyendo a España. Así podría indicarlo la creación de un espacio económico común entre Rusia, Bielorrusia y Kazajstán, que para Putin —en un artículo publicado en Izvestia - supondría el primer paso hacia una futura "Unión Eurasiática" de los miembros de la CEI, casi equivalente en cuanto a su nivel de integración a la $\mathrm{UE}^{32}$. Por otra parte, el anuncio de la candidatura del actual primer ministro a la presidencia ha coincidido también con una visita a China; lo cual podría deberse a una prioridad del vector asiático como contrapeso del vector occidental en la política exterior rusa.

Sin embargo, varias razones hacen dudar de que este cambio en la cúpula del poder ruso vaya a suponer per se una ruptura con la etapa actual, a no ser que entren en juego otros factores. En primer lugar, si el fin de la presidencia de Medvedev en 2012 se debe a que como opina la mayoría de los analistas- Putin ha gozado como primer ministro de una influencia igual o mayor que la de aquél en la formulación de las políticas, parece difícil afirmar que el actual momento de relativa distensión con Occidente se haya producido exclusivamente por iniciativa de Medvedev, y no como fruto de un consenso entre ambos. Al mismo tiempo, hay que recordar que no sólo Putin sino también el actual presidente han defendido el derecho de Rusia a defender sus propios intereses incluso frente a gobiernos percibidos como pro-occidentales, como el caso de la guerra ruso-georgiana en agosto de 2008. Las diferencias en las políticas exteriores de ambos líderes han sido en gran parte una cuestión de imagen más que de contenido real, al cultivar Medvedev una apariencia más moderna y dialogante que la de su predecesor; lo cual se ha visto facilitado también por la actitud más receptiva que ha encontrado en Occidente, gracias al reset impulsado por Obama y al progresivo desbloqueo del diálogo con la UE y la OTAN.

Por esta razón, el probable próximo presidente ruso seguirá interesado — como ya lo hizo durante tras su llegada al poder en 2000- en mantener relaciones fluidas con la UE, a quien continúa necesitando como socio comercial e inversor para modernizar y diversificar la economía de su país; en un mundo cada vez más multipolar donde — pese a la retórica acerca de supuestos "bloques" como el BRIC - tanto europeos como rusos perciben con recelo el declive de su poder relativo en comparación con otras potencias emergentes, como China. En consecuencia, España tiene la oportunidad de aprovechar su asociación estratégica con Moscú para actuar como mediadora, fomentando una mayor aproximación ruso-europea mediante la cual ambas partes vean reforzada su influencia internacional; y pese a que esta aproximación no vaya a suponer — ni tiene por qué condicionarse a — una coincidencia de intereses en todos los ámbitos de las respectivas políticas exteriores.

\footnotetext{
32 "Article published in the newspaper Izvestia - A new integration project for Eurasia: The future in the making", 4 de octubre de 2011, en http://premier.gov.ru.
} 